\title{
Hemodynamic Changes in the Breast and Frontal Cortex of Mothers During Breastfeeding
}

\author{
KIMIE TANIMOTO, TAKASHI KUSAKA, TOMOKO NISHIDA, KAYO OGAWA, IKUKO KATO, SONOKO IJICHI, \\ JUNKO MIKAMI, IKUKO SOBUE, KENICHI ISOBE, AND SUSUMU ITOH
}

\author{
School of Nursing [K.T., J.M., I.S.], Maternal Perinatal Center [T.K.], Department of Pediatrics [I.K., S.IJICHI, K.I., S.ITOH], \\ Faculty of Medicine, Kagawa University, Kagawa 761-0793, Japan; Department of Education for Children with Special Needs [T.N.], \\ Faculty of Education, Kagawa University, Kagawa 760-8521, Japan; Faculty of Nursing [K.O.], Shikoku University, Tokushima 771-1192, Japan
}

\begin{abstract}
The objective of this study was to confirm physiological reactions in the breast and brain in mothers during breastfeeding and collect basic objective data, aiming at effective support for breastfeeding. Ten healthy women who were exclusively breastfeeding their babies participated in this study. Changes in the concentration of oxygenated $\mathrm{Hb}$ (oxy $\mathrm{Hb}$ ) and deoxygenated $\mathrm{Hb}$ in the breasts and frontal cortex of these women during breastfeeding lactation were measured using double-channel near-infrared spectroscopy (NIRS). Changes were measured in three conditions: (1) in both breasts; (2) the ipsilateral breast and frontal cortex; and (3) the contralateral breast and frontal cortex. $\mathrm{OxyHb}$ and total $\mathrm{Hb}$ (total $\mathrm{Hb}$ ) levels in the bilateral breasts decreased significantly after the onset of breastfeeding in comparison with prebreastfeeding levels. These two values repeatedly increased and decreased thereafter. In the frontal cortex, regardless of which breast was involved, oxyHb and totalHb levels increased significantly in comparison with prebreastfeeding levels. Similar hemodynamic changes occurred simultaneously in the bilateral breasts during breastfeeding regardless of the feeding or nonfeeding side. Hemodynamic changes were also noted in the frontal cortex, but the reactions in the breast and prefrontal cortex were different and not synchronous, confirming that the physiological circulatory dynamics during breastfeeding vary among organs. (Pediatr Res 70: 400-405, 2011)
\end{abstract}

$\mathrm{T}_{\mathrm{t}}^{\mathrm{h}}, \mathrm{w}$ he World Health Organization (WHO) and United Nations Children's Fund (UNICEF) in the Innocenti Declaration recommend solely nourishing all infants with breast milk for the first 6 mo. Many long- and short-term advantages of breastfeeding for both children and mothers have been reported. Breast milk is rich in secretory $\operatorname{IgA}(1)$ and has been shown to decrease the incidences of atopic diseases in children (2) as well as the risk of many infectious diseases $(3,4)$. In addition, infants fed breast milk showed a higher cognitive development score than those fed formula milk (5). Appropriate support for mothers and infants early in the breastfeeding process is necessary, and, for this, a refined scientific understanding of breastfeeding and lactation is needed $(6,7)$.

Received October 25, 2010; accepted April 25, 2011.

Correspondence: Takashi Kusaka, M.D., Maternal Perinatal Center, Faculty of Medicine Kagawa University, 1750-1 Ikenobe, Miki-cho Kita-gun, Kagawa 761-0793, Japan; e-mail: Kusaka@med.kagawa-u.ac.jp

Supported by the Prioritized Project of Kagawa University Faculty of Medicine, No.9 and grants-in-aid for scientific research (C) no. 20591299, 22591201, 22591202, and 22591203 from the Ministry of Education, Culture, Sports, Science and Technology of Japan, Sanju Alumni Research Grant 21-1, and The Mother and Child Health Foundation, Japan.
In physiological studies on the breast during feeding, intramammary pressure (8) and oxytocin and prolactin as breastfeeding-related hormone secretion in blood samples have been investigated, and sucking stimulation has been found to induce the release of oxytocin from the posterior pituitary as an immediate reflex (9-11). Ramsay et al. (12) also observed dilatation of the milk ducts using ultrasound as a response to the ejection of milk.

Near-infrared spectroscopy (NIRS) is capable of noninvasively evaluating the tissue blood volume and oxygenation state of the brain (13-15) and muscle. It has also been applied to breast tissue (16) and was found to be particularly useful for the screening of breast cancer $(17,18)$. We previously reported that the tissue blood volume significantly decreased after the onset of breastfeeding in the ipsilateral and contralateral breast and then fluctuated cyclically using NIRS (19). We speculated that the signal on sucking stimulation was transmitted to the brain via the medulla oblongata and induced oxytocin release from the pituitary gland, resulting in hemodynamic changes in the breast, and then hypothesized that there is a close relationship between blood volume changes in the breast and those in the brain.

Investigating physiological relationships during breastfeeding can aid understanding of normal responses during breastfeeding. Such knowledge is valuable from a clinical perspective, as it allows clinicians to develop objective breastfeeding support plans that take into account such influences upon the working of the prefrontal cortex as depression and medication. Depressive symptomatology in the postpartum period negatively influences infant-feeding outcomes, and these findings have important clinical implications and support the need for the early identification and treatment of women with depressive symptomatology (20). A study using multichannel NIRS reported that bipolar disorder and major depression were characterized by preserved but delayed and reduced frontal lobe activations (15). Further, investigation of hemodynamic relationships between the breast and brain during lactation will also contribute to early identification and evaluation for

\footnotetext{
Abbreviations: deoxyHb, deoxygenated hemoglobin; NIRS, near-infrared spectroscopy; oxyHb, oxygenated hemoglobin; PDB, point of the biggest difference in the breast; PDF, point of the biggest difference in the frontal cortex; totalHb, total hemoglobin
} 
treatment. In addition, an understanding of physiological responses during breastfeeding can help clinicians distinguish between mothers who establish normal mother-child relationships during breastfeeding from those mothers who do not, thereby enabling clinicians to help mothers establish normal breastfeeding patterns.

In this study, we performed simultaneous measurements in the bilateral breasts or unilateral breast and forehead during breastfeeding using two-channel NIRS and investigated whether: (1) the blood volume changes in the bilateral breasts regardless of feeding from the ipsilateral or contralateral side and (2) breastfeeding induced changes in the frontal cortex hemodynamics.

\section{METHODS}

Participants. The participants were 10 healthy women who were exclusively breastfeeding their babies, meeting the following inclusion criteria: infants were born after 38 wk of gestation, nourished exclusively by breastfeeding, and developed no congenital diseases, at which time the feeding pattern was assumed to have stabilized, while their mothers were healthy and had no history of breast tissue abnormality.

This study was approved in advance by the Ethics Committee of the faculty of Medicine, Kagawa University. Informed consent was obtained by explaining the study method to the women orally and with written documents, and then obtaining written consent from all women to participate in this study.

NIRS setting. Using double-channel NIRS (NIRO-200, Hamamatsu Photonics K.K., Hamamatsu, Japan), changes in the oxygenated $\mathrm{Hb}$ (oxyHb), deoxygenated $\mathrm{Hb}$ (deoxy $\mathrm{Hb})$, and total $\mathrm{Hb}$ (total $\mathrm{Hb}$ ) levels were measured in the breast and frontal cortex. For NIRO-200, three types of laser diode (775, 810 , and $850 \mathrm{~nm}$ ) were used as the light source, and a detector probe received light. The distance between the emitter and detector probes was $4 \mathrm{~cm}$ for both the breast and frontal head, and both probes, forming one set, were covered with black rubber holders (Fig. 1). For analysis, the modified Beer-Lambert Law (MBL) method was used: changes in the oxyHb, deoxyHb, and totalHb levels were calculated from changes in the optical attenuation time at the light receiver. The measurement interval was $1 \mathrm{~s}$. The sum of [oxyHb] and [deoxyHb] was calculated as [totalHb].

Study procedures. Measurement was performed in a quiet, private room. The infant was fed corresponding to his/her appetite. Each mother sat on a chair and probes were attached. Three conditions of measurement were used. In condition $\mathrm{A}$, the probe sets were attached to the bilateral breasts for simultaneous measurement. In condition B, one probe set was attached to the right breast and the other to the right frontal head in mothers who fed from the right breast, for simultaneous measurement in the ipsilateral breast and frontal head. In condition $\mathrm{C}$, one probe set was attached to the right breast and the

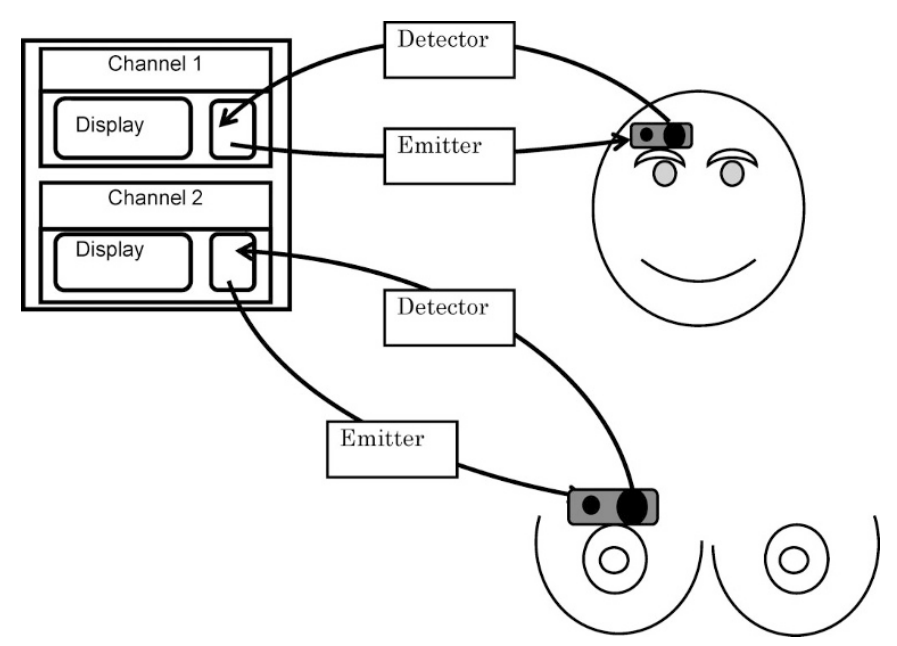

Figure 1. Illustration of probe sets attached to the breast and frontal cortex in conditions B and C. For condition A, the channel 1 probe set was attached to the left breast so as to arrange the detector and emitter on the medial and lateral sides of the body, respectively. other to the right frontal head in mothers who fed from the left breast, for simultaneous measurement of the contralateral breast and frontal head.

The probes covered with rubber holders were applied with tape to a site directly above the areola and directly above the right eyebrow on the frontal head so as to arrange the light detector and emitter on the medial and lateral sides of the body, respectively. Measurement was started before the initiation of feeding and ended more than $60 \mathrm{~s}$ after its completion.

The maternal resting state was measured for more than $60 \mathrm{~s}$ before feeding, during which time the infant was placed in a cot in the same room. The mother then held and fed the baby from the breast on the side she preferred. Mothers were asked to indicate when they sensed milk ejection. Each woman remained in the chair after feeding, and measurement was continued for more than $60 \mathrm{~s}$. Events, such as holding the baby and starting and finishing feeding, were marked on NIRS, and all variables were recorded. A stable environment for the mother and baby was maintained throughout the experiment as much as possible.

Measurement following a different condition was performed on another day when the mother gave consent. Measurement was performed in 10 women: 8 women in condition A (twice for 3 women), 9 in condition B (twice for 3 women), and 8 in condition C (twice for 1 woman).

Data analysis. NIRS data on the breast and frontal head were sampled every other second throughout the measurement period, and the means for $30 \mathrm{~s}$ including the time point described below were calculated. The baseline was defined as the mean for a 30 -s period with small variations before feeding. The time point at which the difference from the baseline [oxyHb] in the breast reached the maximum value after measurement initiation was designated as the point of the biggest difference in the breast (PDB) and that in the frontal cortex as the point of the biggest difference in the frontal cortex (PDF).

To confirm the responses of the frontal head and breast to feeding, differences between the baseline and PDB, and between the baseline and PDF, as well as differences in the parameters between the ipsi- and contralateral breasts were analyzed using the Wilcoxon matched pairs signed rank test. For the confirmation of differences in the characteristics of the mothers and infants among the three study conditions, the Kruskal-Wallis test was used for quantitative and the $\chi^{2}$ test for qualitative variables. All parameters are presented as the means \pm SE. Statistical analysis was performed using SPSS 16.0J (SPSS Japan, Inc.), and the significance level was set at 0.05 .

\section{RESULTS}

Characteristics of participants. The characteristics of the mothers and infants and the number of measurements in each condition are shown in Table 1 . The age of the 10 mothers ranged from 23 to $36 \mathrm{y}$ (mean $\pm \mathrm{SD}: 29.9 \pm 5.5 \mathrm{y}$ ), and the parity was 1 in 6 mothers, 2 in 2, and 3 in 2. One mother gave birth by caesarean section due to prolonged labor. All mothers were right-handed. Infants were born at 38-41 wk of gestation and were aged $8-23 \mathrm{wk}$ at the time of the study. The mean duration of feeding observed was $640 \pm 304 \mathrm{~s}(\mathrm{SD})$. Only feeder breasts in the first measurement were analyzed, and the duration of feeding was $340 \pm 22 \mathrm{~s}$. There were no significant differences in the characteristics of the mothers and infants among conditions $\mathrm{A}, \mathrm{B}$, and $\mathrm{C}$.

Condition A (simultaneous measurement in the bilateral breasts). Figure 2 shows typical changes in [oxyHb], [de$\mathrm{oxyHb}$, and [totalHb] during the period from probe attachment to the bilateral breasts to the completion of feeding. After the onset of feeding, [oxyHb] and [totalHb] decreased in both the ipsilateral and contralateral breasts. These parameters then repeatedly increased and decreased, and markedly decreased after the mother felt the ejection reflex.

Measurement was performed 11 times in eight mothers. The three variables were compared at the ipsilateral and contralateral PDB, and the maximum difference from the baseline [oxy $\mathrm{Hb}$ ] was noted. [oxyHb] and [totalHb] were significantly decreased in the breast tissue at both the ipsilateral and contralateral PDB (Table 2). 
Table 1. Details of mother and infant characteristics

\begin{tabular}{|c|c|c|c|c|c|c|c|c|c|}
\hline \multirow[b]{2}{*}{ ID } & \multicolumn{2}{|c|}{ Mothers } & \multicolumn{4}{|c|}{ Infants } & \multicolumn{3}{|c|}{ Number of measurements } \\
\hline & Age $(y)$ & Parity & Gestational age (wk) & Birth weight (g) & Gender & Age (wk) & Condition A & Condition B & Condition $\mathrm{C}$ \\
\hline 1 & 30 & 1 & 38 & 2832 & $\mathrm{~F}$ & 11 & 1 & 2 & 0 \\
\hline 2 & 35 & 2 & 40 & 3456 & $\mathrm{~F}$ & 23 & 1 & 1 & 1 \\
\hline 3 & 35 & 1 & 41 & 3760 & $\mathrm{~F}$ & 9 & 2 & 1 & 1 \\
\hline 4 & 36 & 3 & 39 & 2956 & $\mathrm{~F}$ & 19 & 1 & 1 & 1 \\
\hline 5 & 23 & 1 & 40 & 3144 & $\mathrm{~F}$ & 21 & 2 & 2 & 2 \\
\hline 6 & 36 & 2 & 39 & 3790 & $\mathrm{M}$ & 8 & 1 & 1 & 1 \\
\hline 7 & 31 & 3 & 39 & 2716 & M & 17 & 1 & 2 & 1 \\
\hline 8 & 24 & 1 & 38 & 2908 & M & 13 & 0 & 1 & 0 \\
\hline 9 & 24 & 1 & 41 & 3748 & $\mathrm{~F}$ & 15 & 2 & 1 & 1 \\
\hline 10 & 25 & 1 & 39 & 2754 & M & 15 & 0 & 0 & 1 \\
\hline
\end{tabular}

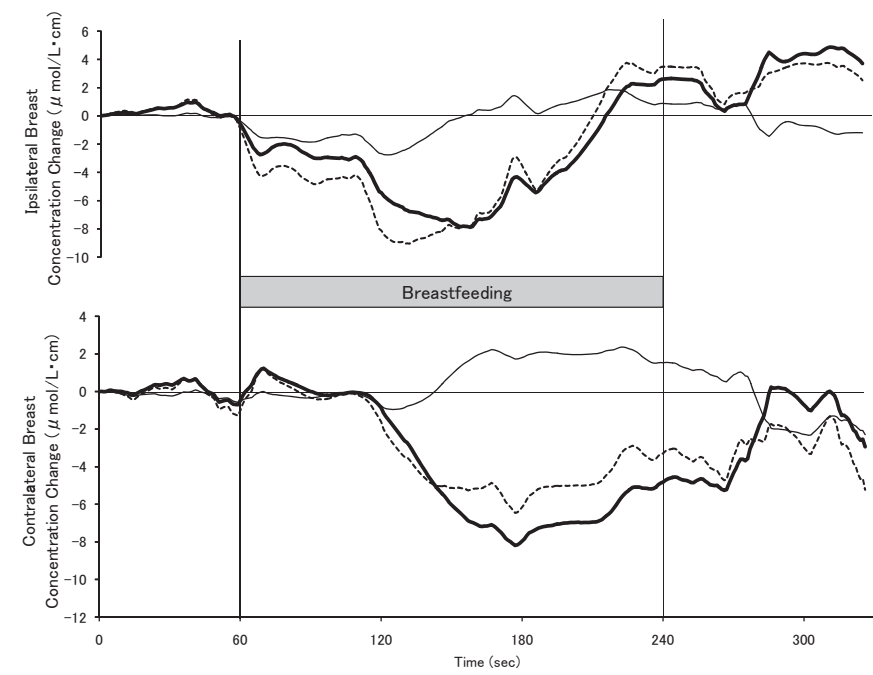

Figure 2. Time-course changes in [oxyHb], [deoxyHb], and [totalHb] in response to feeding stimulation simultaneously measured in the ipsi- (upper) and contralateral (lower) breasts by NIRS. The thick, thin, and dotted lines represent [oxyHb], [deoxyHb], and [totalHb], respectively. Data were smoothed by a gliding average procedure over $6 \mathrm{~s}$. The values of a typical case (condition A) are presented. [oxyHb] and [totalHb] decreased in the bilateral breasts after the start of feeding. [oxyHb] and [total Hb] repeatedly increased and decreased thereafter and then markedly decreased in the bilateral breasts after the mother reported the milk ejection reflex.

The mean changes in [oxyHb], [deoxyHb], and [totalHb] calculated from the differences between the baselines and levels at PDB were $-6.24,-0.07$, and $-6.31 \mu \mathrm{M} \cdot \mathrm{cm}$ in the ipsilateral breast, respectively, and $-3.95,0.08$, and -3.87 $\mu \mathrm{M} \cdot \mathrm{cm}$ in the contralateral breast, respectively. The parameters were compared between the ipsilateral and contralateral breasts to investigate differences associated with the presence or absence of sucking, but no differences were noted in any parameter. The duration of feeding on the feeding side used in the first measurement was $299 \pm 29 \mathrm{~s}$. The time required for [oxyHb] to reach the lowest level after the onset of feeding was $50-149 \mathrm{~s}$ in the ipsilateral and $24-149 \mathrm{~s}$ in the contralateral breast. The lowest level was reached earlier in the ipsilateral breast in four cases and the contralateral breast in seven cases. The time difference in each mother ranged from 0 to $38 \mathrm{~s}$ (mean \pm SD: $17 \pm 14$ ), showing no significant difference between the two sides. [oxyHb] periodically changed on both sides at the same frequency $(5.5 \pm 1.1$ times $)$ over a $66 \pm 8$-s cycle in all mothers. The mothers reported the milk ejection reflex in 8 of 11 measurements. All of them reported one reflex, and $[0 x y H b]$ reached the lowest level immediately after reporting in six.

Condition B (simultaneous measurement in the ipsilateral breast and frontal head). Figure 3 shows typical changes in [oxyHb], [deoxyHb], and [totalHb] during the period from probe attachment to the right frontal head to the completion of feeding from the right breast. Soon after the onset of feeding, [oxyHb] and [totalHb] increased in the frontal head and [deoxyHb] also slightly increased. [oxyHb] and [totalHb] remained high-throughout feeding. [deoxyHb] returned to the baseline level during feeding. [oxyHb], [deoxyHb], and [totalHb] decreased after the onset of feeding in the breast, but [deoxyHb] returned to the baseline level during feeding. $[0 x y H b]$ and [totalHb] increased thereafter and then markedly decreased after the mother reported the feeling of milk ejection. [oxyHb] and [totalHb] returned to the baseline levels while repeating increases and decreases after the completion of feeding.

Measurement was performed 12 times in 9 mothers. The duration of the initial feeding on the ipsilateral side was $354 \pm$ $37 \mathrm{~s}$. The frontal cortex [oxyHb] periodically changed $26.4 \pm$ 3.1 times over a $15 \pm 2$-s cycle. The ipsilateral breast [oxyHb] repeatedly increased and decreased $6.9 \pm 1.4$ times over a $64 \pm 9$-s cycle. The three variables were compared at the time points of the maximum difference from the baseline [oxyHb] (PDF and PDB in the frontal cortex and breast, respectively). [oxyHb] and [totalHb] were significantly higher than the baselines at PDF in the ipsilateral frontal cortex. In contrast, [oxyHb] and [totalHb] simultaneously measured in the ipsilateral breast were significantly lower than the baselines at PDB (Table 2). The differences in the ipsilateral breast [oxyHb], [deoxyHb], and [totalHb] from the baselines were not significant at PDF, but [oxyHb] and [totalHb] in the frontal cortex were significantly higher than the baselines at PDB. The milk ejection reflex was reported in 9 of 12 measurements, and the breast [oxyHb] reached the lowest level immediately after the mother reported the milk ejection reflex in seven.

Condition C (simultaneous measurement in the contralateral breast and frontal head). Probes were attached to the right frontal head and breast in mothers who fed from the left breast. Changes in [oxyHb], [deoxyHb], and [totalHb] were similar and cyclical to those in condition B. After feeding, 
Table 2. Results at baseline and PDF of PDB values

\begin{tabular}{|c|c|c|c|}
\hline & Baseline value $(\mu \mathrm{mol} / \mathrm{L} \cdot \mathrm{cm})$ & PDF or PDB value $(\mu \mathrm{mol} / \mathrm{L} \cdot \mathrm{cm})$ & $p^{*}$ \\
\hline \multicolumn{4}{|c|}{ Condition A $(\mathrm{n}=11)$} \\
\hline \multicolumn{4}{|c|}{ Ipsilateral breast } \\
\hline [oxyHb] & $1.73 \pm 0.94(-2.67$ to 7.63$)$ & $-4.51 \pm 1.42(-13.51$ to 3.61$)$ & 0.004 \\
\hline [totalHb] & $1.54 \pm 0.94(-2.43$ to 6.50$)$ & $-4.77 \pm 1.71(-14.16$ to 3.40$)$ & 0.006 \\
\hline \multicolumn{4}{|c|}{ Contralateral breast } \\
\hline [oxyHb] & $1.48 \pm 0.52(-0.50$ to 4.85$)$ & $-3.18 \pm 1.54(-13.99$ to 5.44$)$ & 0.008 \\
\hline \multicolumn{4}{|c|}{ Condition B $(\mathrm{n}=12)$} \\
\hline \multicolumn{4}{|c|}{ Ipsilateral forehead } \\
\hline [oxyHb] & $-0.53 \pm 0.43(-2.91$ to 2.06$)$ & $1.81 \pm 0.58(-2.16$ to 4.70$)$ & 0.002 \\
\hline [deoxyHb] & $-0.14 \pm 0.23(-1.79$ to 1.00$)$ & $-0.54 \pm 0.29(-2.04$ to 1.21$)$ & 0.099 \\
\hline$[$ total $\mathrm{Hb}]$ & $-0.67 \pm 0.54(-3.91$ to 2.85$)$ & $2.06 \pm 0.80(-1.87$ to 6.78$)$ & 0.003 \\
\hline \multicolumn{4}{|c|}{ Ipsilateral breast } \\
\hline \multicolumn{4}{|c|}{ Contralateral forehead } \\
\hline$[\mathrm{oxyHb}]$ & $-0.41 \pm 0.43(-2.26$ to 1.22$)$ & $1.79 \pm 0.87(-1.06$ to 6.62$)$ & 0.008 \\
\hline [deoxyHb] & $-0.54 \pm 0.33(-2.27$ to 0.49$)$ & $-0.68 \pm 0.37(-2.76$ to 0.90$)$ & 0.214 \\
\hline$[$ total $\mathrm{Hb}]$ & $-0.96 \pm 0.67(-4.53$ to 1.03$)$ & $1.86 \pm 0.97(-1.81$ to 6.43$)$ & 0.008 \\
\hline \multicolumn{4}{|c|}{ Contralateral breast } \\
\hline$[\mathrm{oxyHb}]$ & $-0.15 \pm 0.73(-3.20$ to 2.83$)$ & $-5.32 \pm 1.94(-17.94$ to -0.06$)$ & 0.011 \\
\hline [deoxyHb] & $-0.48 \pm 0.36(-2.30$ to 0.86$)$ & $-1.70 \pm 1.23(-9.84$ to 1.80$)$ & 0.374 \\
\hline [totalHb] & $-0.64 \pm 1.03(-5.03$ to 2.82$)$ & $-7.02 \pm 2.83(-22.35$ to 1.74$)$ & 0.015 \\
\hline
\end{tabular}

* Significance of differences between the baseline and PDF or PDB value (Wilcoxon matched pairs signed rank test).

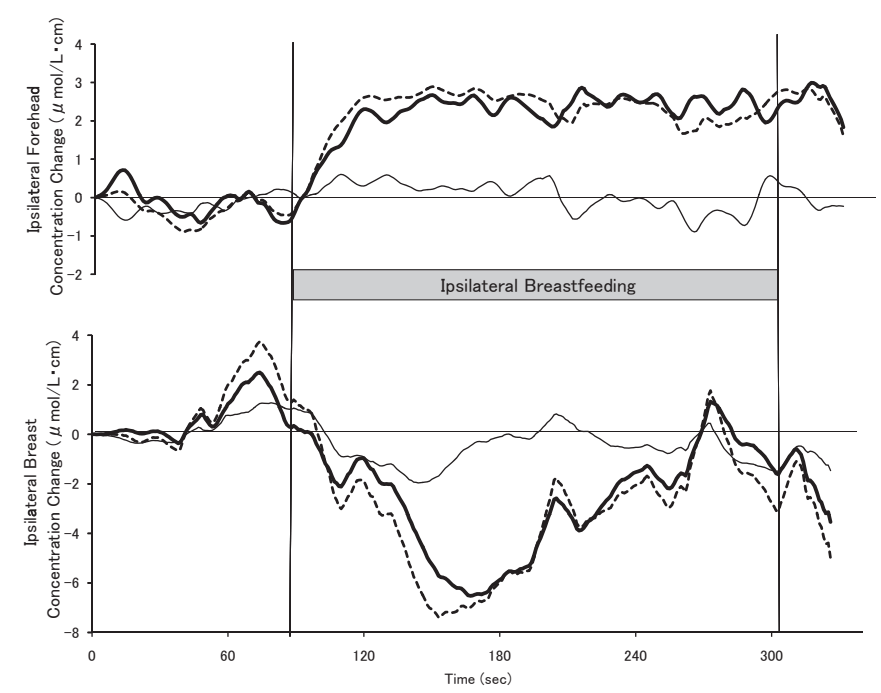

Figure 3. Time-course changes in [oxyHb], [deoxyHb], and [totalHb] in response to feeding stimulation simultaneously measured in the ipsilateral frontal cortex (upper) and breast (lower) by NIRS. The thick, thin, and dotted lines represent [oxyHb], [deoxyHb], and [totalHb], respectively. Data were smoothed by a gliding average procedure over $6 \mathrm{~s}$. The values of a typical case (condition B) are presented. [oxyHb] and [totalHb] increased in the frontal cortex and decreased in the breast after feeding began. After the mother reported the milk ejection reflex, breast [oxyHb] and [total Hb] further decreased markedly, but no change was noted in the levels in the frontal cortex.

[oxyHb] and [totalHb] increased in the head and decreased in the breast and then repeatedly increased and decreased several times.

Measurement was performed nine times in eight mothers. The duration of feeding on the contralateral side was $373 \pm$
$50 \mathrm{~s}$. The contralateral frontal head and breast [oxyHb] periodically changed $27.4 \pm 4.2$ and $8.7 \pm 1.9$ times, respectively. The lengths of one cycle were $14 \pm 1$ and $52 \pm 6 \mathrm{~s}$ in the contralateral head and breast, respectively. The three variables were compared at PDF and PDB. [oxyHb] and [totalHb] in the contralateral frontal head were significantly higher than the baselines at PDF. In contrast, [oxyHb] and [totalHb] simultaneously measured in the contralateral breast were significantly lower than the baselines at PDB (Table 2). The differences in [oxyHb], [deoxyHb], and [totalHb] from the baselines in the contralateral breast were not significant at PDF, but [oxyHb] and [total $\mathrm{Hb}$ ] in the frontal head at PDB were significantly higher than the baselines, as occurred in condition $\mathrm{B}$.

Baselines and times required to reach $P D F$ and $P D B$. The mean time required for the frontal head [oxyHb] to reach the peak was $76 \mathrm{~s}$ in condition B and $77 \mathrm{~s}$ in condition C, showing no significant difference between the conditions. The frontal cortex [oxyHb] peaked before starting feeding in two and one mother in conditions $\mathrm{B}$ and $\mathrm{C}$, respectively. The breast $[\mathrm{oxyHb}]$ reached the lowest level after the onset of feeding in all mothers.

\section{DISCUSSION}

Feeding-induced changes in the oxygenation state in the breast. After the onset of feeding, the blood volume decreased in both the ipsilateral and contralateral breasts. These parameters then repeatedly increased and decreased, and markedly decreased after the mother felt the reflex.

The oxyHb level reduced during breastfeeding in both breasts regardless of whether feeding took place on the ipsi- 
lateral or contralateral side. The time required to reach the first lowest level was not significantly different between the ipsilateral and contralateral sides. After that, the oxyHb level increased and decreased repeatedly. In our previous study (19), we confirmed that the oxyHb level was significantly reduced by sucking stimulation using single-channel NIRS. This phenomenon was also observed in this study using double-channel NIRS, confirming that it occurred simultaneously in both breasts regardless of whether feeding took place on the ipsilateral or contralateral side. This phenomenon is supported by a study which measured milk ejection simultaneously in each breast during double pumping (21), and it was assumed that milk ejection occurs at the same time in both breasts due to the systematic release of oxytocin.

The stimulus of nipple sucking by a baby generates sensory impulses that are transmitted to the spinal cord and then to the secretory oxytocinergic neurons in the hypothalamus. The oxytocinergic neurons induce a massive release of oxytocin from the posterior pituitary into the blood stream $(22,23)$ and oxytocin is carried to the lactating breasts. Oxytocin induces myoepithelial cell contraction around the mammary gland, thus ejecting milk into the duct system (24). Our results show that the blood volume in the measurement region directly above the areola of the nipple decreases after the onset of breastfeeding and then increases and decreases in a repetitive fashion that is congruent with milk ejection. The duration of the cyclic changes is congruent with duct dilation measured using ultrasound (12) and also with the duration of oxytocin pulses in the blood $(10,11)$. The drop in the blood volume may be due to the mechanical compression of blood vessels surrounding the alveoli during myoepithelial cell contraction. Janbu et al. (25) measured the lateral thoracic artery during feeding using pulsed Doppler and observed that the blood flow rate started to decrease before the mother sensed the milk ejection reflex, and then the reflex occurred. They also reported that this phenomenon was induced by the i.v. injection of oxytocin, and the changes in the blood flow rate reflected the reduction of the blood volume. Ramsay et al. (12) measured the lactiferous duct diameter on the contralateral side during feeding and observed that the diameter increased when the mother sensed the milk ejection reflex and the pattern of sucking by her baby changed. These findings support our study results.

Breastfeeding-induced hemodynamic changes in the frontal head. Breastfeeding increased the blood volume in the frontal head. The region measured by NIRS at the frontal head included the cerebral frontal cortex (26). The frontal cortex is considered to play an important role in cognitive control (27), and blood volume changes in this region reflect the modulation of cerebral functional activity $(28,29)$. Our data revealed that breastfeeding increased the blood volume in the frontal cortex in mothers, reflecting activation of the brain. In previous studies using NIRS which confirmed brain responses to fine motor $(30-33)$, gross motor $(34,35)$, visual stimuli (29,36-38), a memorizing task (39), and other cognitive stimulations $(13,26)$, both oxyHb and totalHb levels increased after stimulation. In this study, the oxyHb level increased in the head before the start of feeding in three cases, suggesting that the brain was not activated by sucking stimulation alone. In the breasts that were simultaneously measured, the oxy $\mathrm{Hb}$ level decreased before the onset of feeding in some mothers. These breast responses may be due to the action of oxytocin. Lucas et al. (10) and McNeilly et al. (11) also confirmed oxytocin secretion before the onset of feeding by measuring the blood oxytocin level, showing that milk ejection in humans is induced by emotional factors, such as a baby crying and preparation for feeding. Because oxytocin also induces systemic vasodilatation, emotional responses of the mother before the onset of feeding may have induced oxytocin secretion, dilating blood vessels and increasing the blood volume in the frontal cortex. Although blood volume changes in the breast and brain were not synchronous, the increased blood volume in the brain may have resulted from emotional frontal cortical responses of the mother in anticipation of feeding, which also induced oxytocin secretion, resulting in the dilatation of blood vessels in the breast. Further studies are required to assess the relationship between the volume of milk secretion and hemodynamic reactions in the breast and brain and establish an objective evaluation method to support breastfeeding. Furthermore, we are also planning to compare cases in which breastfeeding is difficult due to psychoneurotic diseases with normal cases and apply the study results to establish a breastfeeding support method.

In conclusion, the blood volume in the bilateral breasts decreased significantly after the onset of breastfeeding and repeatedly increased and decreased thereafter, but the cerebral blood volume in the frontal cortex of the mother increased. Hemodynamic changes also occurred in the frontal cortex, but the reactions in the breast and frontal cortex were different and not synchronous, confirming that the physiological circulatory dynamics during breastfeeding vary among organs.

Acknowledgments. We are grateful to the women and infants for their participation and cooperation in this study. We are also grateful to Hamamatsu Photonics for providing us with materials and Professor Dorothy Powell of Duke University for invaluable suggestions.

\section{REFERENCES}

1. Campbell C 1996 Breastfeeding and health in the Western world. Br J Gen Pract 46:613-617

2. Saarinen UM, Kajosaari M 1995 Breastfeeding as prophylaxis against atopic disease: prospective follow-up study until 17 years old. Lancet 346:1065-1069

3. Dewey KG, Heinig MJ, Nommsen-Rivers LA 1995 Differences in morbidity between breast-fed and formula-fed infants. J Pediatr 126:696-702

4. Duncan B, Ey J, Holberg CJ, Wright AL, Martinez FD, Taussig LM 1993 Exclusive breast-feeding for at least 4 months protects against otitis media. Pediatrics 91:867872

5. Anderson JW, Johnstone BM, Remley DT 1999 Breast-feeding and cognitive development: a meta-analysis. Am J Clin Nutr 70:525-535

6. Brown LP, Bair AH, Meier PP 2003 Does federal funding for breastfeeding research target our national health objectives? Pediatrics 111:e360-e364

7. Gartner LM, Morton J, Lawrence RA, Naylor AJ, O'Hare D, Schanler RJ, Eidelman AI; American Academy of Pediatrics Section on Breastfeeding 2005 Breastfeeding and the use of human milk. Pediatrics 115:496-506

8. Luther EC, Arballo JC, Sala NL, Cordero Funes JC 1974 Suckling pressure in humans: relationship to oxytocin-reproducing reflex milk ejection. J Appl Physiol 36:350-353

9. Weitzman RE, Leake RD, Rubin RT, Fisher DA 1980 The effect of nursing on neurohypophyseal hormone and prolactin secretion in human subjects. J Clin Endocrinol Metab 51:836-839

10. Lucas A, Drewett RB, Mitchell MD 1980 Breast-feeding and plasma oxytocin concentrations. BMJ 281:834-835 
11. McNeilly AS, Robinson IC, Houston MJ, Howie PW 1983 Release of oxytocin and prolactin in response to suckling. BMJ (Clin Res Ed) 286:257-259

12. Ramsay DT, Kent JC, Owens RA, Hartmann PE 2004 Ultrasound imaging of milk ejection in the breast of lactating women. Pediatrics 113:361-367

13. Schroeter ML, Zysset S, Kupka T, Kruggel F, Yves von Cramon D 2002 Nearinfrared spectroscopy can detect brain activity during a color-word matching Stroop task in an event-related design. Hum Brain Mapp 17:61-71

14. Rasmussen P, Dawson EA, Nybo L, van Lieshout JJ, Secher NH, Gjedde A 2007 Capillary-oxygenation-level-dependent near-infrared spectrometry in frontal lobe of humans. J Cereb Blood Flow Metab 27:1082-1093

15. Kameyama M, Fukuda M, Yamagishi Y, Sato T, Uehara T, Ito M, Suto T, Mikuni M 2006 Frontal lobe function in bipolar disorder: a multichannel near-infrared spectroscopy study. Neuroimage 29:172-184

16. Stahel MC, Wolf M, Banos A, Hornung R 2009 Optical properties of the breast during spontaneous and birth control pill-mediated menstrual cycles. Lasers Med Sci 24:901-907

17. Tromberg BJ, Shah N, Lanning R, Cerussi A, Espinoza J, Pham T, Svaasand L, Butler J 2000 Non-invasive in vivo characterization of breast tumors using photon migration spectroscopy. Neoplasia 2:26-40

18. Shah N, Cerussi A, Eker C, Espinoza J, Butler J, Fishkin J, Hornung R, Tromberg B 2001 Noninvasive functional optical spectroscopy of human breast tissue. Proc Natl Acad Sci USA 98:4420-4425

19. Ogawa K, Kusaka T, Tanimoto K, Nishida T, Isobe K, Itoh S 2008 Changes in breast hemodynamics in breastfeeding mothers. J Hum Lact 24:415-421

20. Dennis CL, McQueen K 2009 The relationship between infant-feeding outcomes and postpartum depression: A qualitative systematic review. Pediatrics 123:e736-e751

21. Prime DK, Geddes DT, Spatz DL, Robert M, Trengove NJ, Hartmann PE 2009 Using milk flow rate to investigate milk ejection in the left and right breasts during simultaneous breast expression in women. Int Breastfeed J 4:10

22. Aono T 1990 [Hormonal control of lactation]. Nippon Sanka Fujinka Gakkai Zasshi 42:867-872

23. Gimpl G, Fahrenholz F 2001 The oxytocin receptor system: structure, function, and regulation. Physiol Rev 81:629-683

24. Ledbetter MS, Muench LK 2003 Postpartum period and lactation physiology. In: Blackburn ST (ed) Maternal Fetal \& Neonatal Physiology: A Clinical Perspective. Saunders, St. Louis, pp 158-179

25. Janbu T, Koss KS, Thoresen M, Wesche J 1985 Blood velocities to the female breast during lactation and following oxytocin injections. J Dev Physiol 7:373-380

26. Hock C, Villringer K, Müller-Spahn F, Wenzel R, Heekeren H, Schuh-Hofer S Hofmann M, Minoshima S, Schwaiger M, Dirnagl U, Villringer A 1997 Decrease in parietal cerebral hemoglobin oxygenation during performance of a verbal fluency task in patients with Alzheimer's disease monitored by means of near-infrared spectroscopy (NIRS) — correlation with simultaneous rCBF-PET measurements. Brain Res 755:293-303

27. Miller EK, Cohen JD 2001 An integrative theory of prefrontal cortex function. Annu Rev Neurosci 24:167-202

28. Obrig H, Wenzel R, Kohl M, Horst S, Wobst P, Steinbrink J, Thomas F, Villringer A 2000 Near-infrared spectroscopy: does it function in functional activation studies of the adult brain? Int J Psychophysiol 35:125-142

29. Villringer A, Chance B 1997 Non-invasive optical spectroscopy and imaging of human brain function. Trends Neurosci 20:435-442

30. Strangman G, Culver JP, Thompson JH, Boas DA 2002 A quantitative comparison of simultaneous BOLD fMRI and NIRS recordings during functional brain activation. Neuroimage 17:719-731

31. Obrig H, Hirth C, Junge-Hülsing JG, Döge C, Wolf T, Dirnagl U, Villringer A 1996 Cerebral oxygenation changes in response to motor stimulation. J Appl Physiol 81:1174-1183

32. Mehagnoul-Schipper DJ, van der Kallen BF, Colier WN, van der Sluijs MC, van Erning LJ, Thijssen HO, Oeseburg B, Hoefnagels WH, Jansen RW 2002 Simultaneous measurements of cerebral oxygenation changes during brain activation by near-infrared spectroscopy and functional magnetic resonance imaging in healthy young and elderly subjects. Hum Brain Mapp 16:14-23

33. Kleinschmidt A, Obrig H, Requardt M, Merboldt KD, Dirnagl U, Villringer A, Frahm J 1996 Simultaneous recording of cerebral blood oxygenation changes during human brain activation by magnetic resonance imaging and near-infrared spectroscopy. J Cereb Blood Flow Metab 16:817-826

34. Rupp T, Perrey S 2008 Prefrontal cortex oxygenation and neuromuscular responses to exhaustive exercise. Eur J Appl Physiol 102:153-163

35. Suzuki M, Miyai I, Ono T, Oda I, Konishi I, Kochiyama T, Kubota K 2004 Prefrontal and premotor cortices are involved in adapting walking and running speed on the treadmill: an optical imaging study. Neuroimage 23:1020-1026

36. Heekeren HR, Obrig H, Wenzel R, Eberle K, Ruben J, Villringer K, Kurth R, Villringer A 1997 Cerebral haemoglobin oxygenation during sustained visual stimulation-a near-infrared spectroscopy study. Philos Trans R Soc Lond B Biol Sci 352:743-750

37. Yang H, Zhou Z, Liu Y, Ruan Z, Gong H, Luo Q, Lu Z 2007 Gender difference in hemodynamic responses of prefrontal area to emotional stress by near-infrared spectroscopy. Behav Brain Res 178:172-176

38. Herrmann MJ, Ehlis AC, Fallgatter AJ 2003 Prefrontal activation through task requirements of emotional induction measured with NIRS. Biol Psychol 64:255-263

39. Hoshi Y, Tsou BH, Billock VA, Tanosaki M, Iguchi Y, Shimada M, Shinba T, Yamada Y, Oda I 2003 Spatiotemporal characteristics of hemodynamic changes in the human lateral prefrontal cortex during working memory tasks. Neuroimage 20:1493-1504 